\title{
O papel do (a) Psicólogo (a) na Unidade Básica de Saúde sob uma Perspectiva da Psicologia da Saúde
}

\author{
The Psychologist's Role in the Basic Health Unit from the Health Psychology Perspective \\ Denize Bernardo dos Santos ${ }^{\dagger}$ Maria Helena Pinheiro Gomes ${ }^{\dagger}$, Bárbara Batista Silveira ${ }^{*}$
}

Como citar esse artigo. DOS SANTOS, D.B; GOMES, M.H.P; SILVEIRA, B.B. O papel do (a) Psicólogo (a) na Unidade Básica de Saúde sob uma Perspectiva da Psicologia da Saúde. Revista Mosaico, v.11, n.1, p. 88-92, 2020.

\author{
Resumo
}

Este artigo tem como objetivo trazer a discussão das práticas da Psicologia da Saúde dentro da Unidade Básica de Saúde (UBS). Para tanto, foi realizada uma pesquisa através da busca ativa de informações em livros, revistas e artigos acadêmicos nas bases de dados SCIELO e PEPSIC, entre os meses de setembro de 2019 e janeiro de 2020. A partir do levantamento da literatura revisada, evidenciamos a grande necessidade da atuação do (a) psicólogo (a) na Unidade Básica de Saúde. A Psicologia na UBS é voltada para o acolhimento e a escuta terapêutica, e tem como proposta oferecer uma importante contribuição na abordagem contextualizada e integral dos indivíduos, familiares e toda a comunidade assistida. Diferenciando-se de uma prática curativa e individualizante, as ações estão voltadas para a promoção da autonomia, conscientização e empoderamento, visando à transformação social e melhora na qualidade de vida dentro do processo saúde-doença.

Palavras-chave: Psicólogo. Psicologia da Saúde. Unidade Básica da Saúde.

\begin{abstract}
This article aims to bring the discussion of Health Psychology practices within the Basic Health Unit (UBS). To this end, a research was carried out through the active search for information in books, magazines and academic articles in the SCIELO and PEPSIC databases, between the months of September 2019 and January 2020. From the survey of the reviewed literature, we highlight the great need for the psychologist's performance in the Basic Health Unit. Psychology at the UBS is focused on welcoming and listening to therapy, and aims to offer an important contribution to the contextualized and comprehensive approach of individuals, family members and the entire assisted community. Differentiating from a curative and individualizing practice, the actions are aimed at promoting autonomy, awareness and empowerment, aiming at social transformation and improving the quality of life within the health-disease process.

Keywords: Psychologist. Health Psychology. Basic Health Unit.
\end{abstract}

\section{Introdução}

No ano de 1986, ocorreu, em nosso país, a $8^{\text {a }}$ Conferência Nacional de Saúde, cujo tema foi "Saúde como Direito de Todos e Dever do Estado", trazendo uma mudança na concepção clássica de atenção à saúde, que era norteada por fundamentos assistencialistas e curativos, centrada na figura do médico e nos hospitais. Neste contexto, foi criado o SUS (Sistema Único de Saúde), regulamentado pela lei 8.080 de 19 de setembro de 1990. (CARVALHO, 2013)

O SUS compreende um conjunto de ações e serviços de saúde, prestados por órgãos e instituições públicas federais, estaduais e municipais, da Administração direta e indireta e das fundações mantidas pelo Poder Público. É regido por alguns princípios e diretrizes que são: universalidade, integralidade, equidade, regionalização e hierarquização, descentralização e comando único e participação popular. (VASCONCELOS; PASHE, 2006)

Para garantir um melhor funcionamento do SUS, ele é articulado de acordo com a complexidade do caso, sendo dividido em quatro níveis: 1) Atenção básica; além do atendimento, visa a realizar promoção, prevenção e recuperação da saúde, é um trabalho voltado também para as famílias. 2) Atenção secundária; a doença já identificada no paciente requer um acompanhamento mais específico. 3) A Atenção terciária; é para paciente com caso mais grave, precisando de internação para melhor tratamento. 4) A Reabilitação; apesar do paciente ter feito todos os procedimentos ele ainda precisa de acompanhamento. (CARVALHO, 2013)

A atenção primária é constituída pelas Unidades

\footnotetext{
Afiliação dos autores:

${ }^{\dagger}$ Graduanda de Psicologia, Universidade de Vassouras, Vassouras, RJ, Brasil

* Professora Titular do Curso de Psicologia da Universidade de Vassouras, Vassouras, RJ, Brasil

* Email de correspondencia: barbie.silveira@hotmail.com
} 
Básicas de Saúde (UBS) e Equipes de Atenção Básica. A UBS é o contato preferencial dos usuários, a principal porta de entrada e centro de comunicação com toda a Rede de Atenção à Saúde. Objetiva promover a saúde, a prevenção de agravos, o diagnóstico, o tratamento, a reabilitação, a redução de danos, desenvolvendo uma atenção integral aos sujeitos. Assim, potencializa-se a melhora nas condições de saúde e autonomia dos indivíduos, e nos determinantes e condicionantes de saúde das coletividades (ALMEIDA, 2011).

O (a) psicólogo (a) da saúde ao trabalhar nesses dispositivos oferecidos pelo SUS, principalmente nas UBS, tem como função a educação para a saúde, a promoção de conhecimento e informações para a comunidade, para as famílias, auxiliando o paciente a entender o processo de tratamento e acompanhar o processo de procedimentos médicos envolvidos. De modo geral, seja em atendimento individual ou em grupo, suas ações buscam desenvolver a autonomia dos sujeitos e das coletividades frente ao processo saúdedoença, bem como procurar estabelecer possibilidades crescentes de saídas coletivas e solidárias para problemas apresentados (BOING; CREPALDI; MORÉ, 2009).

Diante do exposto acima, surge o seguinte questionamento: Como se dá a prática do (a) psicólogo (a) na Unidade Básica de Saúde?

A fim de responder tal questionamento este artigo de revisão da literatura especializada tem como objetivo abordar as práticas de trabalho da psicologia da saúde e destacar a importância da inserção do (a) psicólogo (a) na Unidade Básica de Saúde.

Para tanto, foi realizada uma pesquisa através da busca ativa de informações em livros, revistas e artigos acadêmicos nas bases de dados SCIELO e PEPSIC, entre os meses de setembro de 2019 e janeiro de 2020.

A escolha do referido tema justifica-se mediante a tantas diversidades de dispositivos que o SUS oferece, sendo a prática do (a) psicólogo (a) de suma importância dentro das UBS como forma de compreender as variáveis psicológicas sobre a manutenção da saúde, o desenvolvimento de doenças e seus comportamentos associados, dando relevância à manutenção e a promoção da saúde e a prevenção de doença.

\section{A Psicologia da Saúde}

Historicamente, a origem da Psicologia da Saúde, ocorreu no ano de 1970 através da American Psychological Association (APA) com um grupo de psicólogos (as) interessado no trabalho do âmbito da saúde, para buscar compreender o processo saúdedoença. (CASTRO; BORNHOLDT, 2004)

Segundo Martins e Rocha Júnior (2001), a Psicologia da Saúde surge então da necessidade de promover e de pensar o processo saúde-doença como um fenômeno social. Ainda, de acordo com os autores supracitados, o interesse dos psicólogos no contexto da saúde, se deu pela saída desses profissionais das clínicas privadas, contribuindo assim, na inserção desses profissionais na área pública.

Complementando, Castro e Bornholdt (2004) inferem que a Psicologia da Saúde em seu principal objetivo, vai além da compreensão de como os fatores biológicos e comportamentais influenciam no processo saúde-doença, considerando também, os fatores sociais.

No Brasil, a Psicologia tem sido inserida nas políticas de saúde a partir do processo de implantação do Sistema Único de Saúde (SUS). A ciência psicológica integra-se ao campo da saúde no momento em que seu campo de compreensão, transposto das ciências físico-naturais, em que as leis da natureza explicavam o fenômeno humano, encontra-se com as relações que os humanos travam consigo mesmos e com os outros, escapando às leis da natureza. (FOUCAULT, 2002)

Segundo Foucault (2002), o crescimento da Psicologia da Saúde pode ser atribuído a três fatores:

a) à evidência do aumento de doenças e da mortalidade, causadas pelo estilo de vida;

b) ao fortalecimento da filosofia dos países industrializados de que os indivíduos são responsáveis por sua própria saúde;

c) ao aumento da discordância com o modelo médico e sua dominância, na atenção à saúde.

Neste contexto, podemos afirmar que a Psicologia da Saúde pode ser vista como um campo da Psicologia que nasce para dar soluções aos problemas de saúde que ultrapassam o campo biológico. Sebastiani (2003) destaca algumas tendências para essa área, tais como: integração de modelos teóricos, modificação de crenças a atitudes com relação às enfermidades, a participação individual e comunitária nas questões de saúde.

\section{A Unidade Básica de Saúde (UBS)}

No Brasil, a partir da Constituição de 1988, a saúde passa a ser um direito para todos e dever do Estado. Em 1990, o SUS é regulamentado através da Lei $n^{0} 8.080 / 1990$, que visa à promoção, a proteção e a recuperação em saúde, através de princípios como a universalidade, a gratuidade, a integralidade e a descentralização. (PAIM, 2009)

As Unidades Básicas de Saúde (UBS) surgiram na década de 1980 no contexto de organização dos serviços, como possibilidade de maior eficácia de tratamento (resolutividade nas questões referentes à saúde). Com a responsabilidade de uma determinada área geográfica, às Unidades cabiam as ações básicas de promoção, prevenção e recuperação, utilizando-se, quando necessário, da referência e contra-referência 
aos outros níveis de atenção, segundo a complexidade considerada em cada caso. (CARVALHO, 2003)

Segundo Carvalho (2003) a UBS é o contato preferencial dos usuários, a principal porta de entrada e centro de comunicação com toda a Rede de Atenção à Saúde. É instalada perto de onde as pessoas moram, trabalham, estudam e vivem e, com isso, desempenha um papel central na garantia de acesso à população e uma atenção à saúde de qualidade.

As atividades realizadas na UBS são voltadas aos atendimentos de atenção primária em saúde, destinadas à comunidade na área de abrangência, constituindose como a principal porta de entrada e centro de comunicação com toda a Rede de Atenção à Saúde. Entre suas principais atribuições estão a promoção e proteção à saúde, diagnóstico, tratamento, reabilitação, redução de danos e manutenção da saúde, visando desenvolver uma atenção integral à população atendida. (BOING; CREPALDI; MORÉ, 2009)

\section{Atuação do (a) Psicólogo (a) na Unidade Básica de Saúde}

Segundo Rocha Júnior (1991) a profissão de psicólogo (a) surgiu em 1962, com a criação da Lei 4.119 de 27/08/1962. Desde seu surgimento até os dias de hoje a Psicologia vem sendo reconhecida em diferentes espaços de atuação, para atender as diversas demandas da sociedade.

A inserção dos (as) psicólogos (as) no âmbito da UBS teve seu início marcado por desafios e algumas dificuldades devido à adaptação do seu modo de trabalho de acordo com aqueles que seriam atendidos e em refletir sobre uma ação diferente daquela que estes profissionais vinham desenvolvendo. Segundo Casseb (2011), o (a) profissional psicólogo (a) que trabalha na área da saúde, deve conhecer o contexto em que vai atuar. Assim, o ambiente é quase sempre determinante nos procedimentos que poderão ser utilizados e, evidentemente, é determinante também nos padrões comportamentais de adoecer, ficar saudável ou melhorar a qualidade de vida.

Geralmente, o psicólogo trabalha no próprio contexto onde a situação/comportamento ocorre; assim, conhecimento sobre epidemiologia, fatores psicossociais de vulnerabilidade para doenças físicas, habilidades de relacionamento interpessoal, familiaridade com outras áreas de conhecimento, como Medicina, Enfermagem, Fisioterapia, Nutrição, entre outras, são importantes e necessárias para a atuação profissional do (a) psicólogo (a) da saúde (CASSEB, 2011).

Evidenciam Bernardes e Guareschi (2010), que os cenários de atuação do psicólogo na UBS devem apontar para os seguintes campos: acolhimento, clínica psicossocial, territorialização, inserção comunitária, trabalho com grupos, fortalecimento dos processos de participação popular e controle social, fortalecimento do trabalho em equipe, apoio institucional, matriciamento de saúde mental, educação em saúde e trabalho intersetorial, entre outros.

Complementando, os (as) psicólogos (as) que atuam na Atenção Básica nas UBS devem receber e ouvir todas as pessoas que procuram seus serviços, de modo universal, de fácil acesso e sem diferenciações excludentes, e a partir daí construir respostas para suas demandas e necessidades. (CREPOP, 2019)

A população atendida pelos (as) psicólogos (as) e demais profissionais é definida e delimitada pela Política da Atenção Básica de Saúde, e é bastante diversificada, organizada de acordo com programas, objetivos e pela territorialização. Nesse sentido, o (a) psicólogo (a) da Atenção Básica tem papel mais ativo, pois tem como atividade não somente atender à demanda da UBS, mas também ir ao encontro da comunidade visando um trabalho educativo de promoção à saúde, realizado em conjunto com a equipe da Unidade, por meio de projetos (caminhadas, grupos, festas etc.) que envolvam seus residentes. (CREPOP, 2010)

Desta forma, podemos inferir que a população atendida pelos (as) psicólogos (as) compreende:

- Crianças

- Jovens

- Adultos

- Idosos

- Familiares dos (as) usuários (as)

- Comunidade

Segundo CREPOP (2019) além dessa população, também são realizadas ações com os (as) profissionais da área de Educação, Assistência Social, Conselhos Tutelares, Judiciário, as equipes do PSF e do Nasf, bem como estagiários (as) de faculdades ou cursos de extensão que desenvolvem atividades na Atenção Básica à Saúde.

\section{Ações Desenvolvidas pelos (as) Psicólogos} (as)

Aanálise da prática de trabalho na UBS indica uma diversidade de ações e diferentes formas de intervenção. Os (as) psicólogos (as) podem realizar diversas ações no dia a dia, que buscam atender a diferentes demandas da UBS, ampliar o seu trabalho e, ainda, adequá-lo a novas realidades e demandas, tais como: o atendimento individual, grupal, o grupo operativo, as palestras nas escolas, igrejas e espaços comunitários. Essas atividades são desenvolvidas nas unidades de saúde e nos espaços comunitários. (CREPOP, 2019)

Segundo o CREPOP (2019) em relação à prática específica do psicólogo, a atuação está voltada 
para o exercício da clínica individual e grupal, tendo como principais recursos o "acolhimento" e a "escuta terapêutica". Desta forma, as atividades específicas dos psicólogos na UBS são identificar, fazer entrevistas, triagem, palestras de prevenção e orientação, realizar grupos, atuar no planejamento familiar e fazer psicoterapia individual e grupal.

Neste contexto, o acolhimento se coloca na porta de entrada do serviço como uma prática que, para além de considerar o sujeito que procura o serviço, com suas condições de vida, requer também a postura inclusiva, com relação a outros tipos de sofrimento que possam ser relativos a processos de marginalização, exclusão ou violências. (CREPOP, 2019)

O(a)psicólogo(a)naAtenção Básicanormalmente é a porta de entrada para todos os problemas relacionados à saúde mental, quase todo tipo de queixa chega à UBS e é encaminhada pelos diferentes profissionais ao psicólogo (CREPOP, 2010). De acordo com Lima (2005) o psicólogo buscar promover e contribuir com a saúde de algumas formas, tais como:

- Incluindo outros profissionais da saúde, no desenvolvimento de práticas referentes aos problemas como DST/HIV/AIDS;

- Identificando os problemas que requerem atenção prioritária na comunidade;

- Executando atividades a partir de temas significativos para o mundo do adolescente e do perfil socioeconômico e epidemiológico do público-alvo;

- Realizando grupos com adolescentes ou idosos, de acordo com as demandas recebidas; reuniões e ações com escolas, com assuntos atuais das comunidades (visando prevenção).

Nesta perspectiva, o (a) psicólogo (a) da saúde precisa ser um profissional que tenha um olhar social, tanto em suas práticas, quanto em sua formação, para que assim, suas contribuições sejam produtivas.

A atuação do (a) psicólogo (a) na UBS tem o intuito de oferecer uma importante contribuição na compreensão contextualizada e integral dos indivíduos, familiares e de toda a comunidade atendida. Porém, ainda há muito a ser feito para que este profissional tenha êxito no seu desempenho, regulamentando seu espaço.

\section{Considerações Finais}

Neste estudo, a partir do levantamento da literatura revisada, foi possível perceber as contribuições da Psicologia e a importância da atuação do (a) psicólogo (a) na Unidade Básica de Saúde.

A Psicologia da Saúde tem como objetivo compreender como os fatores biológicos, comportamentais e sociais influenciam no processo saúde-doença. Desta forma, a atuação do (a) psicólogo (a) na UBS é voltada para o acolhimento e a escuta terapêutica, e tem como proposta oferecer uma significativa contribuição na compreensão contextualizada e integral dos indivíduos, familiares e toda a comunidade assistida.

É importante ressaltar que a atuação do (a) psicólogo (a) na UBS não se limita a uma prática curativa e individualizante, mas abrange ações que promovem autonomia, conscientização e empoderamento, visando à transformação social da comunidade.

Por fim, espera-se que o presente estudo desperte interesse e incentive a realização de novas pesquisas na área da Psicologia da Saúde, em específico a atuação do (a) psicólogo (a) na Unidade Básica de Saúde. Espera-se também, que os resultados apresentados levem a reflexão e conscientização sobre a necessidade e importância do (a) psicólogo (a) na equipe multiprofissional, sob um ponto de vista interdisciplinar, no contexto da UBS.

\section{Referências}

ALMEIDA, R. A. de; MALAGRIS, L. E. N. A prática da psicologia da saúde. Rev. SBPH, Rio de Janeiro, v. 14, n. 2, p. 183-202, dez. 2011. Disponível em http://pepsic.bvsalud.org/scielo.php?script=sci arttext\&pid=S15160858 2011000200012\&lng=pt\&nrm=iso. Acesso em: $01 \overline{\text { set. }} 2019$.

BERNARDES A. G.; GUARESCHI N. M. F. Práticas psicológicas: enfrentamentos entre saúde pública e saúde coletiva. Estudos de Psicologia. Natal. 2010. Disponível em: http://www.scielo.br/ pdf/epsic/v15n3/ a06v15n3.pdf. Acesso em: 01 set.2019

BOING, E.; CREPALDI, M. A.; MORÉ, C. L. O. O. A Epistemologia Sistêmica como Substrato à Atuação do Psicólogo na Atenção Básica. Revista Psicologia Ciência e Profissão, v.29, n. 4, p.828-845, 2009.

CARVALHO, N. M. Os territórios da saúde e a saúde dos territórios: discutindo o processo de territorialização em saúde a partir do caso de um serviço de atenção primária em $\mathrm{POA} / \mathrm{RS}$. Dissertação (Mestrado em Administração) Faculdade de Administração, Universidade Federal do Rio Grande do Sul. 2003.

CARVAlHO, G. A saúde pública no Brasil. Estud. av., São Paulo , v. 27, n. 78, p. 7-26, 2013. Disponível em: http://www.scielo.br/scielo. php?script $=$ sci_arttext\&pid $=\mathrm{S} 010340142013000200002 \& \operatorname{lng}=\mathrm{en} \& \mathrm{nrm}=\mathrm{is}$ o. Acesso em: 05 Setembro de 2019.

CASTRO, E. K.; BORNHOLDT, E. Psicologia da saúde x Psicologia hospitalar: Definições e possibilidades de inserção profissional. Revista Psicologia ciência e profissão, v.24, n.3, Brasília, set. 2004.

CASSEB, M. S. Efeito de três procedimentos de intervenção sobre adesão ao tratamento em adultos com diabetes. Tese. Universidade Federal do Pará. Núcleo de Teoria e Pesquisa do Comportamento. Programa de PósGraduação em Teoria e Pesquisa do Comportamento. Belém .2011.

CREPOP. Centro de Referência Técnica em Psicologia e Políticas Públicas. Práticas profissionais de Psicólogos e Psicólogas na Atenção Básica à Saúde. $1^{\text {a }}$ Edição Brasília, DF. 2010.

Referências Técnicas para Atuação de Psicólogas (os) na Atenção Básica a Saúde. $1^{\text {a }}$ Edição Brasília, DF. 2019.

FOUCAULT, M. A Psicologia de 1850 a 1950. In: Foucault, M. Ditos e escritos I (p. 133-151). Rio de Janeiro: Forense Universitária. 2002.

LIMA, M. Atuação psicológica coletiva: uma trajetória profissional em unidade básica de saúde. Psicologia em Estudo, Maringá, v. 10, n. 3, p. 431440, set./dez. 2005.

MARTINS, D. G.; ROCHA JÚNIOR, A. Psicologia da saúde e o novo paradigma: Novo paradigma? Revista psicologia teoria e prática, São Paulo, 
v. 3, n. 1, maio. 2001.

PAIM, J. S. O que é o SUS. Rio de Janeiro: Fiocruz, 2009.

ROCHA JUNIOR, A. Das discussões em torno da formação em psicologia às diretrizes curriculares. Revista psicologia teoria e prática, v.1, n.2, p. 3-8. 1991.

SEBASTIANI, R. W. Psicologia da Saúde no Brasil: 50 Anos de História. Revista Suma Psicológica, v. 10, nº 1, p. $25-42.2003$.

VASCONCELOS, C. M.; PASHE, D. F. O Sistema Único de Saúde. In: G.

W. S. CAMPOS et. al. Tratado de Saúde Coletiva. São Paulo: Hucitec; Rio de Janeiro: Fiocruz. 2006 